ARTICLE

\title{
Immune tolerance and immunosuppression in solid organ transplantation
}

\author{
C W N Spearman, ${ }^{1}$ FCP (SA), PhD; Z A Barday, ${ }^{2}$ MB ChB, FCP (SA), Cert Nephrology (SA) \\ ${ }^{1}$ Division of Hepatology, Department of Medicine, Faculty of Health Sciences, University of Cape Town, South Africa \\ ${ }^{2}$ Division of Nephrology, Department of Medicine, Faculty of Health Sciences, University of Cape Town, South Africa
}

Corresponding author: Z A Barday (zunaid.barday@uct.ac.za)

\begin{abstract}
Organ transplantation is the treatment of choice for patients with end-stage organ failure. Most of them will require lifelong immunosuppression to prevent both acute and chronic rejection. T-cell recognition of the allograft major histocompatibility complex antigens is the central event initiating cellular rejection of the allograft, and subsequent full T-cell activation requires three signals. Immunosuppressive regimens currently used in clinical practice are nonspecific and target T-cell activation, clonal expansion or differentiation into effector T cells. While these therapeutic regimens have advanced considerably and one-year graft survival figures for most solid organ transplants (SOTs) are $>90 \%$, the long-term graft survival remains fair owing to graft loss from chronic rejection. The 'holy grail' of SOT is therefore the development of a permanent specific immune tolerance against donor allogeneic antigens without the long-term use of immunosuppression.
\end{abstract}

S Afr Med J 2014;104(11):795. DOI:10.7196/SAMJ.8960

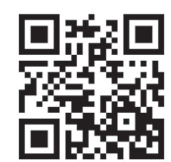

Organ transplantation is the treatment of choice for patients with end-stage organ failure. Most of them will require lifelong immunosuppression to prevent both acute and chronic rejection. According to the Organ Donor Foundation (http://www.odf.org.za), in 2013, 335 solid organ transplants (SOTs) were performed in South Africa (SA). The majority of these were kidney transplants, followed by heart, liver, pancreas and lung transplants. As there are few dedicated transplant centres in SA, physicians and general practitioners are often the primary caregivers and manage transplant recipients as part of shared care with the transplant centre. It is hoped that this review provides insight into the mechanisms of tolerance and the immunosuppressive armamentarium.

T-cell recognition of the allograft major histocompatibility complex antigens is the central event initiating cellular rejection of the allograft, and subsequent full T-cell activation requires three signals (Fig. 1). ${ }^{[1,2]}$ Immunosuppressive regimens currently used in clinical practice are nonspecific and target T-cell activation, clonal expansion or differentiation into effector T cells. While these therapeutic regimens have advanced considerably and one-year graft survival figures for most SOTs are $>90 \%$, long-term graft survival remains poor owing to graft loss from chronic rejection. These immunosuppressive drugs are also associated with significant long-term side-effects, such as opportunistic infections, malignancies, nephrotoxicity, hypertension and diabetes mellitus. Experimental models suggest that induction of tolerance can prevent chronic rejection.

\section{Transplantation tolerance}

The 'holy grail' of SOT is therefore the development of a permanent specific immune tolerance against donor allogeneic antigens without the long-term use of immunosuppression. ${ }^{[2]}$ Clinically, this is known as operational tolerance and is defined as a well-functioning graft lacking histological signs of acute or chronic rejection in the absence of immunosuppression for at least one year in an immunocompetent person capable of responding to infections. ${ }^{[3-5]}$
However, operational tolerance occurs infrequently and is difficult to predict, and incidence varies by allograft type. It occurs in up to $20 \%$ of liver transplant recipients and is much less common in renal transplant recipients, with only isolated case reports of operational tolerance in lung and heart transplant recipients. ${ }^{[3-6]}$

Although the liver is an immunologically privileged organ, operational tolerance is still difficult to achieve shortly after transplantation, but becomes increasingly frequent during the second decade of transplantation. ${ }^{[7]}$ Other clinical factors favouring operational tolerance include paediatric transplantation and livingrelated liver transplantation. ${ }^{[6,7]}$

Operationally tolerant transplant recipients have been shown to exhibit signature transcriptional gene profiles, which differ in liver and kidney transplant recipients. Liver transplant recipients express a high number of natural killer cell genes, while kidney transplant recipients express $\mathrm{B}$-cell regulatory genes. The $\mathrm{T}$ regulatory cell is a common effector cell, as suggested by an increase in FOXP3 expression in operationally tolerant recipients. ${ }^{[6,8]}$

Clinically operational tolerance can be divided into three groups: ${ }^{[5,6]}$ - Spontaneous tolerance in patients who have been non-compliant and discontinued all immunosuppression.

- Planned weaning under medical supervision to reduce side-effects and toxicity of immunosuppression.

- Active application of tolerogenic protocols.

Various experimental tolerogenic protocols based on the mechanisms of central and peripheral tolerance that normally maintain immune homeostasis and self-tolerance have been tested in animal and nonhuman primate models, with only a few entering clinical trials and clinical practice ${ }^{[9]}$ Central tolerance results from the clonal deletion of self-reactive $\mathrm{T}$ cells (death of T cells with T-cell receptors recognising host antigens) during maturation in the thymus. Protocols enhancing central tolerance are based on attempting to establish chimerism, usually microchimerism, which is defined as the presence of donor and recipient cell lineages coexisting within the bone marrow of a myeloconditioned recipient. ${ }^{[10]}$ 


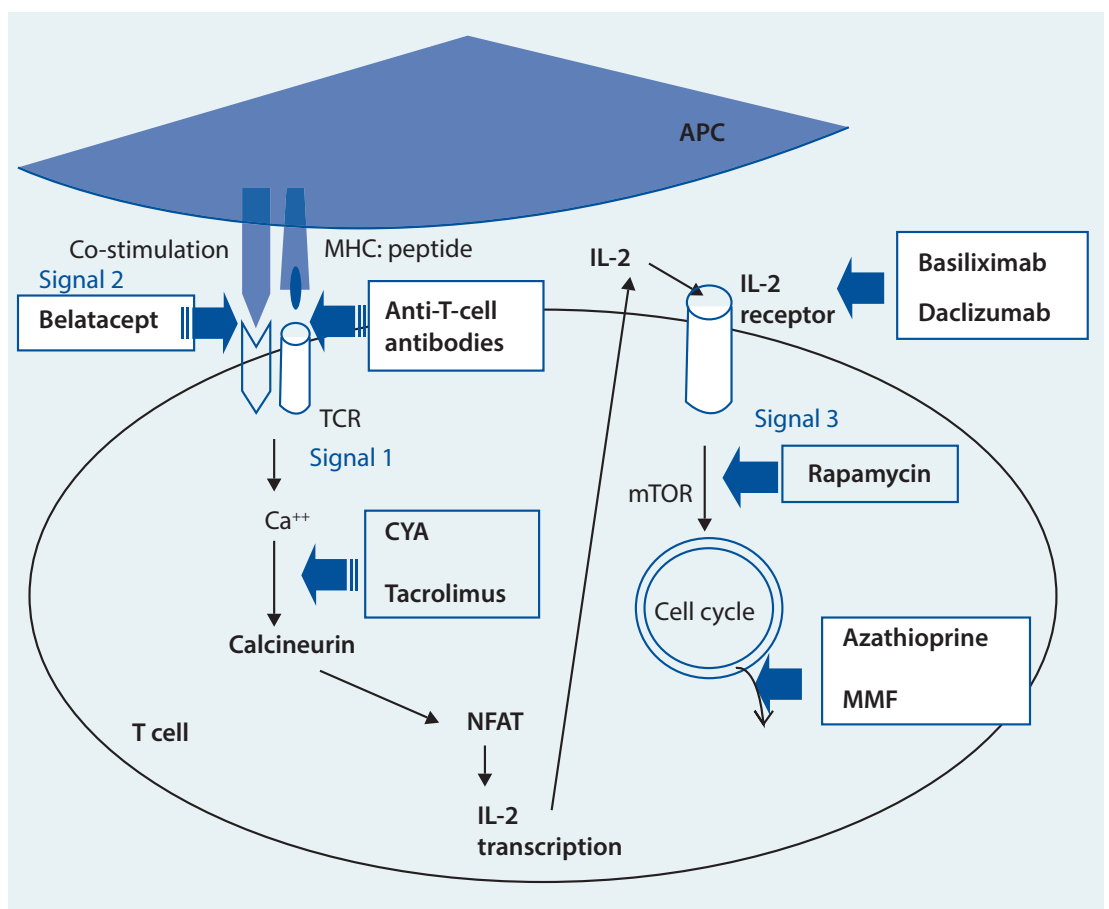

Fig. 1. Targets for current immunosuppressive drugs. Signal 1 is delivered through the T-cell receptor by recognition of peptide antigens presented in the context of major histocompatibility complex molecules on the antigen-presenting cells. This stimulation results in calcineurin activation, a process blocked by cyclosporin A or tacrolimus. Activated calcineurin dephosphorylates the nuclear factor of activated $T$ cells so that it can enter the nucleus and bind to the interleukin-2 (IL-2) promoter. Co-stimulatory signals (signal 2) are necessary for optimal IL-2 gene transcription in the T cell. IL-2-receptor stimulation induces the T cell to enter the cell cycle and proliferate (signal 3); this can be blocked by IL-2-receptor antibodies or by rapamycin, which inhibits signalling induced by IL-2-receptor ligation. By blocking purine synthesis, azathioprine and mycophenolate mophetil interrupt DNA replication and cell proliferation. (APC = antigen-presenting cells; CYA = cyclosporin A; MHC = major histocompatibility complex; $M M F=$ mycophenolate mophetil; $m T O R=$ mammalian target of rapamycin; NFAT = nuclear factor of activated $T$ cells; TCR = T-cell receptor.) (Modified from Golshayan et al. ${ }^{[1]}$ )

Peripheral tolerance involves the suppression or elimination of self-reactive mature T cells in the periphery. This is achieved through inactivation of auto-reactive T-cell clones by inhibitory molecules or suppression by regulatory $\mathrm{T}$ cells (Tregs), with the resulting T-cell inactivation leading to anergy and apoptosis.

Potential protocols enhancing peripheral tolerance include: ${ }^{[1,6,11,12]}$

- Inhibition of alloreactive T-cell responses, including T-cell-depleting strategies, co-stimulatory blockade and interleukin (IL)-2-receptor blockade.

- Inhibition of the humoral response including B-cell depleting therapies (rituximab), blockade of B-cell activating factor (belimumab) and complement protein C5 (eculizumab) production and induction of mature plasma cell apoptosis (bortezomib).

- Anti-cytokine and anti-chemokine therapies preventing homing of activated alloreactive $\mathrm{T}$ cells to the allograft.
- Enhancement of immune regulatory mechanisms to inhibit alloreactive T-cell effector responses via direct ligation or inhibitory cytokine production. This includes infusion of tolerogenic immunomodulatory cells such as expanded regulatory $\mathrm{T}$ cells (Tregs), dendritic cells, macrophages (transplant acceptance-inducing cells) and mesenchymal stromal cells.

\section{Tolerogenic protocols in clinical practice}

Despite success in animal and non-human primate models, very few protocols have been successfully used in clinical practice and usually not as stand-alone therapy. A number of review articles address tolerogenic protocols in detail. ${ }^{[1,6,11,12]}$

Microchimerism. These protocols have involved conditioning of the recipient with thymic irradiation or total lymphoid irradiation plus anti-thymocyte globulin (ATG), followed by the infusion of donor bone marrow. This also increased the number of $\mathrm{CD}^{+} \mathrm{CD}^{+} 5^{+}$Tregs compared with CD4 $\mathrm{T}$ cells and successfully induced tolerance in a small number of kidney transplant recipients.

T-cell depletion. Alemtuzumab (CAMPATH-1H), a humanised anti-CD52 monoclonal antibody (mAb) that depletes $\mathrm{T}, \mathrm{B}$ and natural killer cells and some monocytes, has been shown to reduce the need for immunosuppression. Depletion has been followed by rapid homeostatic proliferation of memory T cells, increased B-cell activating factor and alloantibody production and therefore this is not a stand-alone therapy. Alemtuzumab has been combined with rabbit ATG and rapamycin, which has the added benefit of inducing Tregs and promoting activation-induced cell death of effector T cells.

Co-stimulatory blockade . Co-stimulation (signal 2) is essential for full T-cell activation and differentiation into effector $\mathrm{T}$ cells. Selective targeting of effector $\mathrm{T}$ cells and sparing of Tregs are beneficial. The two main co-stimulatory pathways are CD28/CTLA-4: B7 and the CD154: CD40 pathways.

- CD28/CTLA-4: B7 blockade, i.e. signal 2 blockade. Belatacept - a modified cytotoxic T-lymphocyte antigen 4 (CTLA-4) immunoglobulin with a high affinity for both CD80 (B7-1) and CD86 (B7-2). Although combined blocking of CD80 and CD86 prevents ligation of CTLA-4 and subsequent T-cell activation, this has also inhibited the function of Tregs. Increased risk of post-transplant lymphoproliferative disorders has been documented.

- CD154: CD40 blockade. Administration of a humanised anti-CD154 mAb in clinical trials has been complicated by the development of thromboembolic events.

Immune tolerance remains difficult to achieve and disruption of a single pathway is usually not sufficient to promote tolerance or longterm allograft survival.

\section{Immunosuppression in solid organ transplantation}

Although immune tolerance is desired in SOT, we still rely on immunosuppression to prevent allograft rejection. Immunosuppression has transformed the field of SOT since agents were first used over 50 years ago, and will still be an essential component of the armamentarium for the foreseeable future. ${ }^{[13,14]}$

Most SOT recipients need induction immunosuppression at the time of transplantation (usually an intravenous antibody preparation) and maintenance 
immunosuppression (usually oral). Almost all immunosuppressive drugs are directed against the T cell (Fig. 1) ${ }^{[1,2]}$ for their mechanisms of action.

The guiding principle with immunosuppression in SOT is to achieve a balance between preventing rejection and avoiding side-effects, which include various common and opportunistic infections, malignancies, the 'metabolic syndrome' group of diseases, as well as problems relating to bone structure and marrow function. These are beyond the scope of this article and are not discussed.

\section{Induction agents}

Although not strictly considered part of induction, all patients receive high-dose steroids, usually intravenous (IV) methylprednisolone, at the time of surgery and for $\geq 2$ days thereafter, depending on the graft type and graft function. Most patients should receive induction therapy at the time of surgery and for a few days to a week thereafter. The most commonly used induction agents are:

- IL-2-receptor blockers. Basiliximab is the only currently available drug in this class. This anti-CD25 monoclonal antibody administered on day 0 and day 4 post-transplant, binds CD25 on the IL-2 receptor of T cells (signal 3 in Fig. 1).

- ATG. This polyclonal antibody preparation (Thymoglobuline/ Fresenius ATG) is prepared by injecting human thymic tissue into rabbits, and therefore has the potential to cause serum sickness-like reactions. Being polyclonal, ATG targets multiple molecules and receptors on thymocytes. ATG is considered the most potent induction agent for preventing and treating acute cellular rejection, but also plays a role in preventing antibodymediated rejection. It causes prolonged lymphocyte suppression, increasing the risk of opportunistic infections and post-transplant lymphoproliferative disease. ATG should therefore be reserved for patients at highest immunological risk of rejection, such as those with a high percentage of preformed and, in particular, donorspecific antibodies.

\section{Maintenance immunosuppression}

These drugs are started at the time of transplantation or even before in living-related transplants, and are usually lifelong. They can be divided into five classes:

- Glucocorticosteroids. Most patients continue oral prednisone after the initial pulses of IV methylprednisolone. The starting dose of 20 - $30 \mathrm{mg}$ daily is tapered to $5 \mathrm{mg}$ daily, or one can alternate daily over $1-3$ months, depending on graft function and use of induction therapy (allows for faster tapering) and other agents used in combination. Owing to side-effects, steroids may occasionally be discontinued, but this increases the risk of rejection and should be avoided unless mandatory. The side-effects of steroids are well described and are not different in transplant recipients. Particular problems include new-onset diabetes after transplantation (NODAT) and avascular necrosis of the hips. The mechanism of action of glucocorticosteroids is not fully understood, but the ability of the intracellular glucocorticoid receptor to alter the activity of key immune-modulatory transcription factors plays a key role.

- Anti-metabolites. Azathioprine (AZA) was the first drug in this class and is still widely used. It was first used as an anti-cancer drug, and is now widely used in preventing rejection in transplant recipients and in various autoimmune diseases. It is considered to be the most 'safe' immunosuppressant in pregnancy. Side-effects include hepatotoxicity and marked bone-marrow suppression when used with allopurinol (xanthine oxidase interferes with normal 6-MP breakdown). It is also potentially carcinogenic. The starting dose of AZA ranges from 0.5 to $2 \mathrm{mg} / \mathrm{kg} /$ day, depending on the white cell count, and should be decreased if cytopenias occur.

- Mycophenolate mofetil (MMF) or sodium (MPS) is converted to mycophenolic acid, once absorbed in the gastrointestinal tract (GIT). Because of lower acute rejection rates and more predictable dosing, it is replacing AZA in the transplant setting. Starting doses are MMF $1 \mathrm{~g}$ bd or MPS $720 \mathrm{mg}$ bd, but lower doses may be required depending on the white cell count. Specific indications include AZA intolerability, rejection on AZA administration or use of allopurinol. Mycophenolates commonly have GIT (nausea, ulceration, diarrhoea) and other side-effects, but these may be less with the enteric-coated MPS. Mycophenolates are teratogenic and contraindicated in pregnancy.

- Calcineurin inhibitors (CNIs). Cyclosporin A (CYA) and tacrolimus (TAC) are the only drugs available in this class. They form the cornerstone of immunosuppression, around which the other drugs are added. TAC is a more potent immunosuppressive with a slightly different side-effect profile (more risk of NODAT and neurological side-effects) to CYA (more risk of hypertension, hyperuricaemia, hirsuitism, gingival hypertrophy and hyperlipidaemia). Both are potentially nephrotoxic (especially cyclosporin) and rarely cause haemolytic-uraemic syndrome. The once-daily prolonged-release TAC formulation potentially improves compliance. Monitoring of CNIs is done using trough levels or a 2-hour concentration (C2) for CYA. Higher trough levels are targeted early post-transplant (CYA $150-300 \mathrm{ng} / \mathrm{mL}$, TAC $8-12 \mathrm{ng} / \mathrm{mL}$ ), and lower levels (CYA $50-150 \mathrm{ng} / \mathrm{mL}$, TAC $4-10 \mathrm{ng} / \mathrm{mL}$ ) after 3 - 6 months. Therapy should however be individualised according to graft type and graft function.

- Mammalian target of rapamycin inhibitors (mTORi). The mTORIs are sirolimus (previously called rapamycin) and everolimus. mTORIs are not nephrotoxic, but are not as potent at preventing acute rejection as CNIs. Also called antiproliferative drugs, they have useful anti-cancer properties, and have been used in the treatment of post-transplant lymphoproliferative disorders (PTLDs) and renal cell carcinoma. mTORIs are the only class of immunosuppressives with a decreased risk of cancer. mTORIs delay wound healing and are not usually used in the peri-transplant period everolimus may, however, be combined with low-dose CNI in this setting. They may need to be stopped around the time of other major surgery. Other side-effects include stomatitis, peripheral oedema, hypercholesterolaemia, proteinuria, and rarely interstitial pneumonitis. About one-third of patients do not tolerate mTORIs and the main indications for use are CNI nephrotoxicity and malignancy in a transplant recipient, especially PTLDs.

- Co-stimulatory inhibitors. The only drug in this class is belatacept (CTLA-4-Ig), a CD28 homologue combined with the Fc portion of IgG3. Being an antibody preparation, it is given IV monthly lifelong and allows for the omission of CNIs.

The most common immunosuppressive regimens used are prednisone with CYA-AZA or TAC-MMF. TAC-MMF is considered the more potent combination for preventing rejection, but is more expensive and provokes more opportunistic infections, e.g. cytomegalovirus (CMV), tuberculosis (TB) and BK virus (polyoma virus) nephropathy. 
CMV prophylaxis with valgancyclovir (3 - 6 months), co-trimoxazole prophylaxis for Pneumocystis pneumonia (6 months) and TB isoniazid prophylaxis ( 1 year) are routinely used.

In summary, operational tolerance, although desirable, remains difficult to achieve in the clinical setting. Tolerance biomarkers may help to identify potential transplant recipients who can be weaned

\section{Transplant definitions}

- Allogeneic: From a genetically non-identical donor of the same species

- Types of rejection: Acute rejection is characterised by an intense cellular and humoral immune attack leading to early allograft damage and potential graft loss if not controlled. Chronic rejection is characterised by obliterative vasculopathy and parenchymal fibrosis leading to allograft loss and is the main cause of late allograft failure

- Immunosuppression: Inhibition of the normal immune response to allogeneic antigens that results in rejection of the allograft

- Tolerance: A state of unresponsiveness to allogeneic antigens in the absence of immunosuppression

- Microchimerism: Presence of donor and recipient cell lineages coexisting within the bone marrow of a myelo-conditioned recipient from maintenance immunosuppression, but this should only be attempted with careful monitoring in transplant recipients with stable graft function on minimal immunosuppression without histological evidence of acute or chronic rejection.

\section{References}

1. Golshayan D, Buhler L, Lechler RI, Pascual M. From current immunosuppressive strategies to clinical tolerance of allografts. Transpl Int 2007;20:12-24. [http://dx.doi.org/10.1111/j.14322277.2006.00401.x]

2. Fuchs EJ. Transplantation tolerance: From theory to clinic. Immunol Rev 2014;258:64-79. [http:// dx.doi.org/10.1111/imr.12154

3. Ashton-Chess J, Giral M, Brouard S, Soulillou P. Spontaneous operational tolerance after immunosuppressive drug withdrawal in clinical renal allotransplantation. Transplantation 2007;84:1215-1219. [http://dx.doi.org/10.1097/01.tp.0000290683.54937.1b]

4. Orlando G, Soker S, Wood K. Operational tolerance after liver transplantation. J Hepatol 2009;50:1247 1257. [http://dx.doi.org/10.1016/j.jhep.2009.03.006]

5. Orlando G, Hematti P, Stratta RJ, et al. Clinical operational tolerance after renal transplantation: Current status and future challenges. Ann Surg 2010;252:915-928. [http://dx.doi.org/10.1097/ SLA.0b013e3181f3efb0]

6. Chandrasekharan D, Issa F, Wood KJ. Achieving operational tolerance in transplantation: How can lessons from the clinic inform research directions? Transpl Int 2013;26:576-589. [http://dx.doi. org/10.1111/tri.12081]

7. Levitsky J. Operational tolerance: Past lessons and future prospects. Liver Transpl 2011;17:222-232. [http://dx.doi.org/10.1002/lt.22265]

8. Heidt S, Wood KJ. Biomarkers of operational tolerance in solid organ transplantation. Expert Opin Med Diagn 2012;6(4):281-293. http://dx.doi.org/10.1517/17530059.2012.680019]

Alpodogan $\mathrm{O}$, van den Brink MRM. Immune tolerance and transplantation. Semin Oncol 2012;39(6):629-642. [http://dx.doi.org/10.1053/j.seminoncol.2012.10.001]

10. Sachs DH, Kawai T, Sykes M. Induction of tolerance through mixed chimerism. Cold Spring Harb Perspect Med 2014;4:a015529. [http://dx.doi.org/10.1101/cshperspect.a015529]

1. Page EK, Dar WA, Knechtle SJ. Tolerogenic therapies in transplantation. Front Immunol 2012;3:1-13. [http://dx.doi.org/10.3389/fimmu.2012.00198]

12. Salisbury EM, Game DS, Lechter RI. Transplantation tolerance. Pediatr Nephrol [epub ahead of print, 10 November 2013].

3. Halloran P. Immunosuppressive drugs for kidney transplantation. N Engl J Med 2004;351:2715-2729. [http://dx.doi.org/10.1056/NEJMra033540]

14. Pharmacy Weekly Drug Interactions Newsletter 2009;1(12)7. 